\title{
Psychometric validation of the Turkish Ten-Item Internet Gaming Disorder Test (IGDT-10)
}

\author{
Cuneyt Evren ${ }^{1 *(}$, Bilge Evren $^{2 \oplus}$, Ercan Dalbudak $^{3 \oplus},{\text { Merve } \text { Topcu }^{4 \oplus} \text {, Nilay Kutlu }}^{2 \oplus}$ \\ 'Bakirkoy Training and Research Hospital for Psychiatry Neurology and Neurosurgery, Research, Treatment and Training Center for \\ Alcohol and Substance Dependence (AMATEM), Istanbul - Turkey \\ ${ }^{2}$ Baltalimani State Hospital for Musculoskeletal Disorders, Department of Psychiatry, Istanbul - Turkey \\ ${ }^{3}$ Yuksek Ihtisas University, Faculty of Medicine, Department of Psychiatry, Ankara - Turkey \\ ${ }^{4}$ Cankaya University, Department of Psychology, Ankara - Turkey
}

\begin{abstract}
Objective: The main aims of the current study were to test the factor structure, reliability and validity of the Ten-Item Internet Gaming Disorder Test (IGDT-10), a standardized measure to assess symptoms and prevalence of internet gaming disorder (IGD).

Method: In the present study, participants were assessed with the IGDT-10, the nine-item IGD Scale - Short Form (IGDS9-SF), and the Motives for Online Gaming Questionnaire (MOGQ).

Results: Confirmatory factor analysis showed that the IGDT-10's one-factor structure (i.e., dimensional structure) was satisfactory. The scale was also reliable (i.e., internally consistent with a Cronbach's alpha of 0.79) and showed adequate convergent and criterion-related validity as shown by positive correlations between average daily time spent playing games over the past year and IGDS9-SF and MOGQ scores. By applying the DSM-5 threshold to diagnose IGD (meeting at least five criteria), the incidence of individuals with IGD was found to be $7.4 \%(n=56)$ in the entire sample.

Conclusion: These results demonstrate the Turkish version of the IGDT-10 to be a valid and reliable instrument for determining the magnitude of problems associated with IGD among young adults and for early diagnosis of IGD in clinical environments and comparable studies.
\end{abstract}

Keywords: E-sports, internet gaming disorder, IGDT-10, scale, university students, video gaming, young adults

\section{INTRODUCTION}

Gaming is a common legitimate leisure activity on the Internet that may become pathological for some players when it turns dysfunctional, harming the social, occupational, domestic, academic, and psychological functioning of an individual (1). "Pathological gaming" can generally be defined as ongoing, recurrent, and excessive participation in uncontrollable computer or video games despite related problems $(2,3)$. Accordingly, the Diagnostic and Statistical Manual of Mental Disorders (DSM-5) included "Internet Gaming Disorder" (IGD) as a condition requiring further studies before being comprehensively recognized as a distinct disorder in future versions of the DSM $(4,5)$. According to the DSM-5, IGD is clinically characterized by a "persistent and recurrent use of the Internet to engage in games, often with other players, leading to

How to cite this article: Evren C, Evren B, Dalbudak E, Topcu M, Kutlu N. Psychometric validation of the Turkish Ten-Item Internet Gaming Disorder Test (IGDT-10). Dusunen Adam The Journal of Psychiatry and Neurological Sciences 2020;33:19-28.

Correspondence: Cuneyt Evren, Bakirkoy Training and Research Hospital for Psychiatry Neurology and Neurosurgery, Research, AMATEM, Bakirkoy, Istanbul - Turkey

Phone: +90 21240915 15-21 11 E-mail: cuneytevren@yahoo.com

Received: August 28, 2019; Revised: September 23, 2019; Accepted: September 27, 2019 
clinically significant impairment or distress" (5). Although IGD contains the word "Internet," the DSM5 states that while IGD most often involves excessive participation in particular Internet games, it may also include involvement with computerized offline games (5). The nine IGD criteria resemble those of gambling disorder and substance use disorder (6). They include preoccupation with Internet games, withdrawal symptoms, tolerance, ineffective efforts to regulate involvement in Internet games, loss of interest in prior hobbies, ongoing excessive use of Internet games, deceiving family members, using Internet games to escape, and losing an important relationship, job, education, or career opportunity (5). In order to be diagnosed as IGD, American Psychiatric Association (APA) suggests that the gamer must fulfill at least five of these nine criteria over a 12-month period (5). Furthermore, the current state of research regarding the clinical relevance of gaming, its health burden and the neurobiological similarities to other addictive disorders warrants inclusion of this condition in the International Classification of Diseases, $11^{\text {th }}$ Revision (ICD-11) as a behavioral addiction (7). In addition, the ICD-11 also classifies IGD as a mental disorder (8).

To determine the validity of a diagnostic entity, the establishment of a formal gaming-related diagnosis has been discussed among academics for over three centuries (9-14). Some of the primary arguments against formalizing the disorder are the absence of agreement on the term used, its operational definition (i.e., criteria), its evaluation, the course of the disorder, and the precise characteristics of the problem behavior (9).

There are arguments against the formalization of the disorder $(9,12)$, and APA has not yet recognized IGD as an official clinical entity (4); however, following the initial inclusion of this condition by APA in the DSM-5, several new promising psychometric tools covering the nine IGD criteria have been developed $(5,6,15-21)$. A recent mini-review of the psychometric evaluation of IGD presented several IGD evaluation tools based on the diagnostic criteria defined by APA in the DSM- 5 $(5,22)$ : the IGD Scale (IGDS) (18), the 20-item IGD Test (IGD-20) (19), the nine-item IGDS - Short-Form (IGDS9-SF) (20), the 10-item IGD Test (IGDT-10) (17), the updated Clinical Video Game Addiction Test (23), and the Video Game Dependency Scale (24).

Unfortunately, some of these measures may be lengthy, while others (including some of the short ones) either do not specifically reflect the nine IGD criteria or have adopted the terminology of the DSM-5 items too closely, which may be difficult for gamers to understand and respond to $(12,17)$. Thus, Kiraly et al. (17) developed and psychometrically validated a brief tool for assessing IGD using the definitions suggested in the DSM-5 while adopting a simple, clear, and more consistent wording that adequately reflects the concept of IGD. The IGDT10 (17) is a short screening tool that evaluates IGD as being operationalized in the DSM-5, adopting a concise, clear, and consistent wording that adequately reflects the IGD construct. It was theoretically informed by a debate of specialists. In order to retain high content validity, the nine DSM-5 criteria for IGD were strictly followed, while also taking into consideration the suggestions of Petry et al. (6) concerning item operationalization. Given the complexity of the final IGD criterion ("Has jeopardized or lost a significant relationship, job, or educational or career opportunity because of participation in Internet games"), Kiraly et al. (17) expanded this criterion into two items to prevent using double-barreled questions in a study using a large sample of online gamers; based on original analysis, their approach showed promising psychometric characteristics (12).

The IGDT-10, which was created to be used in largescale studies, has been validated in several languages, and has outstanding psychometric properties and concise characteristics to evaluate IGD in time-limited studies. The IGDT-10 was used in both adolescent and adult samples to evaluate the symptoms and incidence of IGD and has a number of benefits over other comparable instruments. First, it was created directly using the nine IGD criteria identified by DSM-5, making it helpful for clinicians to diagnose IGD by the scores achieved by respondents evaluated with this instrument (12). Second, IGDT-10 can be administered quickly because it includes only ten items, possibly assisting clinicians in crowded clinical environments. Petry et al. (6) suggested that "establishing the psychometric properties of instruments assessing these nine (IGD) criteria should begin using a cross-cultural perspective." Since gaming behavior may vary across cultures, studies examining the psychometric characteristics of IGDT-10 are required in different languages and cultures around the world. This notion is partly supported by studies conducted in different languages, including Chinese (25), Finnish (26), Hungarian, Persian (Farsi), English, French, Norwegian, Czech, and Spanish (Peru) (12).

The comparative advantages of the IGDT-10 over other brief tools, such as IGDS (18) and IGDS9-SF (20), are the clear and user-friendly wording and extensive coverage of all IGD criteria as suggested by DSM-5 (12). Although IGDS (27) and IGDS9-SF $(28,29)$ were 
validated in Turkish previously, these advantages of the IGDT-10 over the other measures motivated the authors to conduct the present study. The objective of this research is therefore to assess the psychometric characteristics (i.e. internal consistency, unidimensionality, construct validity, convergence, and criteria-related validity) of the Turkish version of the IGDT-10 among both professional and nonprofessional gamers. By carrying out the present study and achieving the proposed goals, we hope to make a unique contribution to the advancement of knowledge regarding the cross-cultural implications in IGDevaluation using the new framework developed by APA in the DSM-5. Furthermore, the results reported here may be fruitful for clinicians in need of expanded data on the diagnostic efficacy of the frequently-used IGD evaluation instrument.

\section{METHOD}

\section{Participants and Procedure}

To test the psychometric characteristics of the Turkish IGDT-10, an online survey was performed using a crosssectional design. Initially, data were collected from people who were in the e-mail database of a company located in Istanbul that organizes e-sports tournaments (ESL Turkey Amateur e-sport players) or in the e-mail database of a game development company located in Ankara (Taleworlds Entertainment), as well as Turkishspeaking gamers from gaming forums. Moreover, additional data were collected from Turkish university students in Ankara. People who reported that they do not play any games were excluded from the study.

The research protocol was approved by the Ethics Committee of Cankaya University (Turkey) and the study was confidential and anonymous. All participants gave informed consent after reading the Plain Language Information Statement. The online survey was created using Qualtrics. Participants sampled from Cankaya University who agreed to engage in the research gained bonus credit, which added to their overall grade for particular courses they were assigned.

A total of 980 potential participants started the online survey. In the initial phase of the data collection stage from March 4 to April 12, 370 amateur or professional gamers began the online survey, 323 of whom were included in the study as data from the remaining participants were systematically incomplete. While these respondents had given informed consent, they were excluded from the research because they did not complete the survey. No one in this group marked the option "I never play video games." In the second phase of the data collection stage from April 16 to May 29, 610 university students started the online survey, and 429 of them were included in the study while 35 students did not fully complete the survey and 146 marked the option "I never play video games." Thus, a total of 752 participants were included in the study.

The main sociodemographic characteristics of the sample are shown in Table 1 . The mean age of the sample was 23.09 years $(\mathrm{SD}=5.10$; minimum $=11$, maximum $=60)$. Among these, 519 were male $(69.0 \%)$ and 233 were female (31.0\%). Of the participants, $566(76.6 \%)$ were students, 665 (88.4\%) were single, 504 (67\%) were living with family, and $329(45.8 \%)$ had a romantic relationship. Forty-three of the participants (5.7\%) described themselves as amateur e-sport gamers, whereas 13 (1.7\%) defined themselves as professional e-sport gamers. Among the participants, 640 (85.1\%) reported that they had started gaming before the age of 6,46 (3.7\%) stated that they were involved in gaming more than 42 hours per week $(\mathrm{h} / \mathrm{w})$ (more than 6 hours per day $[\mathrm{h} / \mathrm{d}]), 368$ (48.9\%) reported that they were gaming more than usual on weekends and $215(28.6 \%)$ said that they have problems related with gaming (Table 1).

\section{Measures}

Gaming Time: Categories for weekly gaming time were the following: (1) "less than seven hours weekly (less than one hour a day)," (2) "7-14 hours weekly (1-2 hours per day)," (3) "15-28 hours weekly (2-4 hours per day)," (4) "29-42 hours weekly (4-6 hours per day)," and (5) "more than 42 hours weekly (more than 6 hours per day)."

Ten-Item Internet Gaming Disorder Test (IGDT10): The IGDT-10 evaluates past-year IGD (17), with 10 items comprising the nine diagnostic criteria of IGD based on DSM-5 (5). Respondents reported each statement's frequency ( 0 ="never"; $1=$ "sometimes"; $2=$ "often"). The responses "Never" and "sometimes" were coded as criterion not being met ( 0 points), while "often" was evaluated as criterion met ( 1 point) to reflect the dichotomous structure of the DSM-5 criteria for IGD. Items 9 and 10 were merged during the scoring because both items referred to the final DSM-5 criterion. The response "often" given to either one of the two items or both contributed one point to the scoring. Thus, the composite rating for IGDT-10 ranged from 0 to 9. According to DSM-5 (5), a score of five or more points indicates clinically relevant cases. This threshold was therefore used to identify the percentage of respondents who met the cutoff score on the IGDT-10 (the potential risk group). 
Table 1: Sociodemographic characteristics of the sample

\begin{tabular}{|c|c|c|}
\hline & $\mathbf{n}$ & $\%$ \\
\hline Age, years; mean (SD) minimum-maximum & $23.09(5.10)$ & $11-60$ \\
\hline Male gender (n, \%) & 519 & 69.0 \\
\hline Romantic relationship & 329 & 45.8 \\
\hline \multicolumn{3}{|l|}{ Marital status } \\
\hline Married & 39 & 5.2 \\
\hline Divorced & 8 & 1.1 \\
\hline Single & 665 & 88.4 \\
\hline Other & 40 & 5.3 \\
\hline \multicolumn{3}{|l|}{ Living... } \\
\hline ...alone & 74 & 9.8 \\
\hline ...with partner & 39 & 5.2 \\
\hline ...with roommate & 84 & 11.2 \\
\hline ...with family & 504 & 67.0 \\
\hline ...in dorm & 51 & 6.8 \\
\hline \multicolumn{3}{|l|}{ Employment } \\
\hline Working & 93 & 12.4 \\
\hline Part-time worker & 25 & 3.3 \\
\hline Unemployed & 36 & 4.8 \\
\hline Student & 576 & 76.6 \\
\hline Other & 22 & 2.9 \\
\hline \multicolumn{3}{|l|}{ Type of participant (n, \%) } \\
\hline Professional e-sport gamer* & 13 & 1.7 \\
\hline Amateur e-sport gamer** & 43 & 5.7 \\
\hline Play games for his/her own pleasure and/or follow e-sports & 211 & 28.1 \\
\hline University student and frequently plays games on the Internet & 485 & 64.5 \\
\hline \multicolumn{3}{|l|}{ Time daily spent gaming ${ }^{A}$, hours $(n, \%)$} \\
\hline Less than $7 \mathrm{~h} / \mathrm{w}$ (Less than $1 \mathrm{~h} / \mathrm{d}$ ) & 716 & 57.3 \\
\hline More than 7 hours, less than 14 h/w (More than 1 hour, less than 2 h/d) & 285 & 22.8 \\
\hline More than 15 hours, less than 28 h/w (More than 2 hours, less than 4 h/d) & 148 & 11.8 \\
\hline More than 29 hours, less than $42 \mathrm{~h} / \mathrm{w}$ (More than 4 hours, less than $6 \mathrm{~h} / \mathrm{d}$ ) & 55 & 4.4 \\
\hline More than $42 \mathrm{~h} / \mathrm{w}$ (More than $6 \mathrm{~h} / \mathrm{d}$ ) & 46 & 3.7 \\
\hline Gaming more than usual in weekends & 368 & 48.9 \\
\hline \multicolumn{3}{|l|}{ Age at first gaming (years) } \\
\hline Until age 6 & 205 & 27.3 \\
\hline Ages between 7-12 & 435 & 57.8 \\
\hline Ages between 13-17 & 84 & 11.2 \\
\hline Ages between 18-25 & 13 & 1.7 \\
\hline After age of 25 & 15 & 2.0 \\
\hline Having problems related with gaming & 215 & 28.6 \\
\hline
\end{tabular}

h/w: hours per week, h/d: hours per day, ${ }^{*}$ Regularly receives monthly salary, ${ }^{*}$ Has a team, participates in tournaments and makes money in tournaments, ${ }^{A}$ During last year, SD: Standard deviation

In this study, two Turkish psychiatrists fluent in English translated the IGDT-10 from English to Turkish. The translated version was agreed by these specialists. In order to establish their comparability, the Turkish version of the IGDT-10 was then translated from Turkish to English by a separate translator. The 
final translation was presented to 30 students ( 15 were male and 15 were female) from Cankaya University to determine whether the language was clear and to ensure the scale's face validity.

Internet Gaming Disorder Scale - Short-Form (IGDS9-SF): The IGDS9-SF evaluates the symptoms and severity of IGD and its detrimental impacts by examining online and/or offline gaming activities that occur over a period of 12 months (20). The scale includes nine items that correspond to the nine main criteria of the DSM-5. Answers are given on a five-point Likert-type scale ranging from (1) (never) to (5) (very often), and high scores on the scale translate to a higher level of gaming disorder. The Turkish version of the IGDS9-SF has been used in this study (28); Cronbach's a was 0.89.

Motives for Online Gaming Questionnaire (MOGQ): Online gaming motives were evaluated by the MOGQ, which is a 27 -item self-report scale that measures 7 motives for online gaming (Social, Escape, Competition, Skill Development, Coping, Fantasy, and Recreation) (30). The MOGQ uses a 5-point Likert scale from "never" to "almost always/always," with higher scores indicating higher frequency of the respective motivational dimension. Internal consistencies ranged from 0.79 to 0.90 for all 7 dimensions (30). Coping and escape factors, which are separate factors in the original scale, were found to be a single factor (coping/escape) in the Turkish version; therefore, this version has six factors, not seven. Item 18 was deleted from the Turkish version because it showed a negative loading. Thus, the total number of items was 26 . Internal consistencies for the present sample were excellent, ranging from 0.87 (social) to 0.91 (coping/escape and skill development). The estimation of a six-factor model produced a good fit $\left(\chi^{2}\right)$ $\mathrm{df}=710.5 / 251=2.83$; GFI $=0.929, \mathrm{CFI}=0.971, \mathrm{TLI}=0.962$, and RMSEA=0.049) (31).

\section{Statistical Analysis}

IBM SPSS Statistics Version 20 was used for statistical analyses, with the exception of Confirmatory Factor Analysis (CFA), which was carried out using IBM SPSS Amos. Before the analyses, data cleaning was performed by inspecting cases with serious missing values across the main variables of interest.

The following strategies were used to investigate the psychometric properties of the Turkish IGDT-10: (a) its factorial structure was firstly examined using Exploratory Factor Analysis (EFA) then CFA; (b) convergent and criterion-related validity were determined by estimating Pearson product moment correlation coefficients between the total scores of the IGDT-10, IGDS9-SF,
MOGQ, and the self-reported average daily time spent playing games during last year; (c) internal consistency was assessed using Cronbach's alpha.

\section{RESULTS}

\section{Factor Structure}

To investigate the factor structure and dimensionality of MOGQ, the data collected were used to conduct an initial EFA followed by CFA.

Bartlett's Sphericity Test and Kaiser-Meyer-Olkin (KMO) measurement of sampling adequacy were used to verify the adequacy of the sample size before any further evaluation. Bartlett's Sphericity Test was significant for the MOGQ $\left(\chi^{2}=1343.874, \mathrm{df}=36, \mathrm{p}<0.001\right)$, and the sampling adequacy measure of KMO was acceptable at 0.877. Principal Axis Factoring extraction method with Promax (oblique) rotation on the nine items of the IGDT-10 (items 9 and 10 were condensed) was performed for a preliminary examination of its factorial structure and construct validity. The number of components to be extracted was determined through examination of a scree plot (32) in combination with the conventional Kaiser criterion guideline (all factors with eigenvalues greater than one) (33). Furthermore, the acceptable threshold of items with factor loadings above 0.50 and/or parallel loadings below 0.20 was used to retain items (34). Based on these procedures, the EFA resulted in a one-factor solution for the nine items of the IGDT-10, conforming to the criterion of an Eigenvalue greater than one (3.949). Overall, the total variance accounted for by this component was $43.88 \%$ (Table 2).

The unidimensionality of the Turkish IGDT-10 was then subsequently assessed via CFA with maximum likelihood. In order to evaluate the quality of the model estimated in the CFA, several fit indices were used and the following thresholds adopted: $\chi^{2} / \mathrm{df} \leq 5$, Goodness of Fit Index (GFI), Tucker-Lewis Fit Index (TLI) and Comparative Fit Index (CFI) $>0.90$, and Root Mean Square Error of Approximation (RMSEA) $<0.05$ (3538). The estimation of a unidimensional model produced a good fit $\left(\chi^{2} / \mathrm{df}=43.7 / 25=1.75\right.$; GFI $=0.987$, $\mathrm{CFI}=0.986$, TLI $=0.980$, and RMSEA $=0.032$ ). As seen in Tables 2 and 3, all item-component loadings were statistically significant and within the conventional acceptable threshold of $>0.50$. Thus, results from the EFA and the CFA suggest that the IGDT-10 assesses a unidimensional construct.

\section{Convergent and Criterion-related Validity}

Convergent validity was assessed by correlating the IGDT-10 scores with the scores of two related scales 
Table 2: Summary of the results from the EFA on the Ten-Item Internet Gaming Disorder Test obtained from the whole sample $(\mathbf{n}=\mathbf{7 5 2})$

\begin{tabular}{|c|c|c|c|c|c|c|}
\hline \multirow{3}{*}{ Item $^{a}$} & & & \multicolumn{2}{|c|}{ Factor loadings } & \multirow{2}{*}{$\begin{array}{c}\text { Cronbach's } \\
\text { Alpha if } \\
\text { item } \\
\text { deleted }\end{array}$} & \\
\hline & \multicolumn{2}{|c|}{$\begin{array}{c}\text { Criterion } \\
\text { endorsement }\end{array}$} & Factor $1^{c}$ & $\begin{array}{l}\text { Corrected } \\
\text { item-total } \\
\text { correlation }\end{array}$ & & \\
\hline & $\mathbf{n}$ & (\%) & & & & \\
\hline 1. Preoccupation/salience & 109 & 14.5 & 0.619 & 0.477 & 0.764 & \\
\hline 2. Withdrawal symptoms & 48 & 6.4 & 0.622 & 0.486 & 0.766 & \\
\hline 3. Tolerance & 83 & 11.0 & 0.653 & 0.511 & 0.760 & \\
\hline 4. Loss of control & 67 & 8.9 & 0.478 & 0.358 & 0.779 & \\
\hline 5. Giving up other activities & 80 & 10.6 & 0.613 & 0.476 & 0.764 & \\
\hline 6. Continuation & 141 & 18.8 & 0.715 & 0.583 & 0.747 & \\
\hline 7. Deception & 61 & 8.1 & 0.570 & 0.444 & 0.769 & \\
\hline 8. Escape & 191 & 25.4 & 0.538 & 0.410 & 0.780 & \\
\hline \multirow[t]{3}{*}{ 9. Negative consequences } & 113 & 15.0 & 0.690 & 0.558 & 0.752 & \\
\hline & \multicolumn{2}{|c|}{$\geq 5$ items } & \multicolumn{2}{|c|}{$<5$ items } & & \\
\hline & $\mathbf{n}$ & $\%$ & $\mathbf{n}$ & $\%$ & $\chi^{2}$ & $\mathbf{p}$ \\
\hline Whole sample & 56 & 7.4 & 696 & 92.6 & & \\
\hline Age & & & & & $<0.001$ & 0.983 \\
\hline Lowest to 21 & 21 & 7.1 & 262 & 92.6 & & \\
\hline 22 to highest & 35 & 7.5 & 434 & 92.5 & & \\
\hline Gender & & & & & 11.625 & 0.001 \\
\hline Female $(n=233)$ & 6 & 2.6 & 227 & 97.4 & & \\
\hline Male $(n=519)$ & 50 & 9.6 & 469 & 90.4 & & \\
\hline Groups & & & & & 24.687 & $<0.001$ \\
\hline Gamers (n=267) & 37 & 13.9 & 230 & 86.1 & & \\
\hline University students ( $\mathrm{n}=485)$ & 19 & 3.9 & 466 & 96.1 & & \\
\hline Eigenvalue & & & 3.949 & & & \\
\hline Variance $\%^{\mathbf{b}}$ & & & 43.88 & & & \\
\hline Mean \pm SD & & & $1.19 \pm 1.82$ & & & \\
\hline Cronbach's a (10 items, 3 response options) & & & 0.85 & & & \\
\hline Cronbach's a (9 items, 2 response options) & & & 0.79 & & & \\
\hline
\end{tabular}

a: Full description of items was omitted from the table for the sake of clarity, ${ }^{b}$ : Percentage of the total variance explained, ${ }^{c}$ : Only one factor could be extracted from the Exploratory Factor Analysis (EFA), SD: Standard deviation

(i.e., the IGDS9-SF and MOGQ), and criterion-related validity was evaluated through examination of the correlation between the IGDT-10 scores and selfreported average daily time spent gaming during the last year. The correlation between the IGDT-10 and the IGDS9-SF $(\mathrm{r}=0.710, \mathrm{p}<0.001)$ was moderate. The correlation between the IGDT-10 and the MOGQ dimensions (Coping/escape: $\mathrm{r}=0.491, \mathrm{p}<0.001$; recreation: $r=0.301, p<0.001$; fantasy: $r=0.423, p<0.001$; skill development: $\mathrm{r}=0.393, \mathrm{p}<0.001$; social: $\mathrm{r}=0.459$, $\mathrm{p}<0.001$; competition: $\mathrm{r}=0.402, \mathrm{p}<0.001$ ) were mild and statistically significant. Moreover, this result was also consistent with the association between the IGDT-10 scores and self-reported average daily time spent on gaming during the last year $(\mathrm{r}=0.463, \mathrm{p}<0.001)$ (Table 4). Overall, these results demonstrate positive correlations among the variables of interest in the expected direction according to the underlying theory, thus supporting the validity of the Turkish IGDT-10.

\section{Internal Consistency Reliability}

As an indicator for the reliability of the Turkish IGDS9SF, Cronbach's alpha coefficient was high both for the 10 items, 3 response options $(\alpha=0.85)$ and the 9 items, 2 
Table 3: Summary of CFA results of factor loadings, Cronbach's alpha and inter-item correlations obtained from the nine items of the IGDS9-SF

\begin{tabular}{|c|c|c|c|c|c|c|c|c|c|}
\hline \multirow[t]{2}{*}{ Item } & \multirow[t]{2}{*}{$\begin{array}{l}\text { Factor } \\
\text { loadings }\end{array}$} & \multicolumn{8}{|c|}{ Inter-item correlations } \\
\hline & & 2 & 3 & 4 & 5 & 6 & 7 & 8 & 9 \\
\hline 1. Preoccupation/salience & 0.569 & 0.356 & 0.349 & 0.123 & 0.323 & 0.402 & 0.251 & 0.246 & 0.313 \\
\hline 2. Withdrawal symptoms & 0.514 & & 0.429 & 0.205 & 0.316 & 0.334 & 0.221 & 0.260 & 0.286 \\
\hline 3. Tolerance & 0.551 & & & 0.203 & 0.333 & 0.364 & 0.253 & 0.272 & 0.339 \\
\hline 4. Loss of control & 0.435 & & & & 0.210 & 0.256 & 0.283 & 0.214 & 0.326 \\
\hline 5. Giving up other activities & 0.542 & & & & & 0.365 & 0.229 & 0.235 & 0.350 \\
\hline 6. Continuation & 0.678 & & & & & & 0.344 & 0.299 & 0.465 \\
\hline 7. Deception & 0.503 & & & & & & & 0.252 & 0.366 \\
\hline 8. Escape & 0.458 & & & & & & & & 0.285 \\
\hline 9. Negative consequences & 0.645 & & & & & & & & - \\
\hline
\end{tabular}

All factor loadings and item-item Pearson correlations were statistically significant ( $p<0.001)$, CFA: Confirmatory Factor Analysis, IGDS9-SF: The nine-item Internet Gaming Disorder Scale - Short Form

Table 4: Correlations between IGDT-10 and IGDS9-SF, average gaming time and MOGQ

\begin{tabular}{lc} 
& IGDT-10* \\
\hline IGDS9-SF & 0.710 \\
Average gaming time** & 0.463 \\
MOGQ & \\
$\quad$ Coping/Escape & 0.491 \\
Recreation & 0.301 \\
Fantasy & 0.423 \\
Skill Development & 0.393 \\
Social & 0.459 \\
Competition & 0.402 \\
\hline
\end{tabular}

*9 items, 2 response options, ${ }^{* *}$ Spearman correlation, all the other correlations are Pearson correlations. All the correlations are significant at the level of $p<0.001$, MOGQ: Motives for Online Gaming Questionnaire, IGDT-10: Ten-Item Internet Gaming Disorder Test, IGDS9-SF: Internet Gaming Disorder Scale-Short Form

response options $(\alpha=0.79)$ (Table 3). Moreover, Cronbach's alpha did not increase by deleting any of the nine items of the scale. Furthermore, item-total correlations for the IGDT-10 were significant, ranging between 0.358 (item 4 [loss of control]) and 0.583 (item 6 [continuation]) (Table 2). Finally, inter-item correlations ranged between 0.123 (between item 1 [preoccupation/salience] and item 4 [loss of control]) and 0.465 (between item 6 [continuation] and item 9 [negative consequences]) (Table 3 ).

\section{Prevalence Rates of Internet Gaming Disorder}

Participants fulfilling at least five of the nine IGD criteria in this study were operationally defined as possibly qualifying for a positive IGD diagnosis according to the diagnosis recommendation given by the APA $(4,6)$. Based on the approach utilized by previous research using the IGDT-10 $(12,17,25,26)$, participants' answers to the IGDT-10 items as (1) "often" were operationalized as meeting a criterion. Thus, the rate of potential IGD was about $7.4 \%(n=56)$ for the whole sample. This rate was higher among males $(9.6 \%, \mathrm{n}=50)$ than females $(2.6 \%, \mathrm{n}=6)$, and was higher among gamers $(13.9 \%, \mathrm{n}=37)$ than university students $(3.9 \%, \mathrm{n}=19)$ (Table 2).

Consistent with these findings, the average time spent daily on gaming during the last year was significantly greater for all male participants than female participants $\left(\chi^{2}=142.872, \mathrm{df}=4, \mathrm{p}<0.001\right)$, and those involved in e-sports spent significantly more daily time gaming during the last year than university students $\left(\chi^{2}=125.224, \mathrm{df}=4, \mathrm{p}<0.001\right)$.

Finally, age at first gaming was negatively correlated with IGD symptom severity $(r=-0.15, p<0.001)$ in Spearman correlation analysis.

\section{DISCUSSION}

The main aim of the current study was to evaluate the psychometric properties of the Turkish IGDT-10. According to this aim, the IGDT-10 was tested in a cross-sectional study using an online survey to recruit Turkish university students and amateur or professional online gamers. The results of the present study support the validity and reliability of the IGDT-10.

EFA and CFA found a single-factor solution for the IGDT-10, further supporting the one-dimensional factor structure of the IGDT-10 found in previous 
studies $(12,17,25,26)$. The findings of the EFA and CFA produced statistically relevant and comparatively high factor loadings, further showing that all items were appropriate indicators of the IGD construct and that the scale has appropriate psychometric characteristics in addition to a strong factor structure.

In the original study, the IGDT-10 showed adequate reliability (Cronbach's alpha of 0.68 ) (17), whereas it was 0.85 for the Chinese version (25) and 0.87 for the Finnish version (26). Other languages also exhibited adequate internal consistency (12). Cronbach's alpha values ranged between 0.77 (French-speaking and English-speaking) to 0.86 (Persian [Farsi]) for the 10 items, 3 response options, whereas it ranged between 0.62 (French-speaking) to 0.75 (Persian [Farsi]) for the 9 items, 2 response options (12). Compared to these Cronbach's alphas, the Turkish version reached similar results regarding the reliability of the IGDT-10 (Cronbach's alpha of 0.85 for the 10 items, 3 response options and 0.79 for the 9 items, 2 response options). A powerful correlation with the Problematic Online Gaming Questionnaire (15) was found in the original IGDT-10 validation study, a measure evaluating a similar structure, and both tools were mildly linked with weekly gaming time, supporting the construct validity of the IGDT-10 (17). Similarly, in the current research, the anticipated positive correlations occurring between the IGDT-10 and all the associated measures supported criterion-related and convergent validity. The convergent validity of the scale was shown by the positive correlations between the IGDT-10 and the IGDS9-SF and the MOGQ, whereas the criterionrelated validity of the scale was stated by the positive correlation with the average daily time spent gaming in the last year. Overall, higher mean results on the IGDT10 indicate higher average daily time spent on games and higher IGD severity and gaming motivation. The IGDT-10 provided a valid and reliable measure of IGD with good diagnostic accuracy that can be used for research and diagnostic purposes among young adult male and female gamers. Overall, previous validity studies for the IGDT-10 $(12,17,25,26)$ were corroborated by the findings of this research.

The prevalence of IGD among adolescent gamers in Taiwan was $3.1 \%$ (25), whereas it was $1.3 \%$ among Finnish vocational school students (26). A crosscultural validation study reported that the proportions of each sample that met the cutoff score on the IGDT10 varied between $1.61 \%$ (Norwegian) and $4.48 \%$ (English-speaking) in the individual samples, except for the Spanish (Peruvian) sample (13.44\%) (12). Thus, rates of IGD according to the cutoff score on the IGDT10 ranged between $1.3 \%$ and $13.44 \%$ in different cultures. In the present study, the prevalence of those potentially meeting the criteria of a positive IGD diagnosis among the whole sample was $7.4 \%(n=56)$. When only those involved in e-sports $(n=267)$ were considered, $13.9 \%(n=37)$ of the sample was classified as potentially IGD positive, while among the rest of the participants, who considered themselves as university students playing games, 3.9\% were classified as IGDpositive $(n=19)$. Similarly, those engaged in e-sports, whether as an amateur or as a professional, had more severe IGD symptoms and spent more average daily gaming time over the past year than students at university. These results are consistent with our previous study $(27,39)$. This rate was higher among males $(9.6 \%, n=50)$ than females $(2.6 \%, n=6)$. Finally, according to the results reported by previous studies $(27,40)$, men appeared to be more involved in gaming behaviors compared to women as they displayed more severe symptoms of IGD and on average spent more daily time on gaming over the past year. Consistent with this observation, in the validation study of the Finnish version of IGDT-10, the male gender was related with higher IGDT-10 scores (26).

The present study has some limitations. First, using an online survey excludes individuals who have no Internet access. Therefore, these results may not be extrapolated to respondents who, due to the absence of Internet access, mostly play offline games. Second, for face validity, the scale was administered only to university students, which do not represent the total sample. Third, since all respondents were self-selected, it is not possible to generalize the current results directly to the general population. Fourth, the research may also be limited by the fact that all information was gathered using self-report questionnaires, a technique that is subject to well-known biases such as social desirability biases, short-term recall biases, etc. Fifth, participants were not assessed for an IGD diagnosis using a gold standard. This research was therefore unable to determine sensitivity and specificity of IGDT-10 in the detection of IGD. Future studies could benefit from replicating these results among people clinically diagnosed with IGD, which would be desirable considering the present scarcity of data from clinical samples.

Despite these possible limitations, the results of the validity and reliability tests for the Turkish IGDT-10 were found to be comparable to prior study findings. The results presented confirm that the Turkish version 
of IGDT-10 with a one-dimensional structure is a valid and reliable IGD screening instrument for evaluating the symptoms and incidence of IGD among young adults. These results support the use of the IGDT-10 for making early diagnoses and for other relevant studies investigating excessive and addictive playing of video games. It may be hoped that the current study will facilitate research into gaming addiction in Turkish-speaking communities, thereby promoting research into culture-specific variables and, at the same time, facilitating a national and international consensus to define video game addiction using the IGD framework.

\begin{tabular}{|c|c|c|}
\hline \multicolumn{2}{|c|}{ Contribution Categories } & \multirow{2}{*}{$\begin{array}{l}\text { Author Initials } \\
\text { C.E., B.E., E.D. }\end{array}$} \\
\hline \multirow{3}{*}{ Category 1} & Concept/Design & \\
\hline & Data acquisition & M.T., N.K. \\
\hline & Data analysis/Interpretation & C.E. \\
\hline \multirow{2}{*}{ Category 2} & Drafting manuscript & C.E., B.E., E.D. \\
\hline & Critical revision of manuscript & C.E. \\
\hline Category 3 & Final approval and accountability & C.E., B.E., E.D., M.T., N.K. \\
\hline \multirow{2}{*}{ Other } & Technical or material support & C.E., M.T., N.K. \\
\hline & Supervision & C.E. \\
\hline
\end{tabular}

Ethics Committee Approval: The research protocol was approved by the Cankaya University (Turkey) Ethical Committee and the study was confidential and anonymous.

Informed Consent: All participants gave informed consent after reading the Plain Language Information Statement.

Peer-review: Externally peer-reviewed.

Conflict of Interest: All authors declare that they have no conflict of interest.

Financial Disclosure: There is no financial support.

\section{REFERENCES}

1. Gentile DA, Choo H, Liau A, Sim T, Li D, Fung D, et al. Pathological video game use among youth: a two-year longitudinal study. Pediatrics 2011; 127:e319-e329.

2. Griffiths MA. A 'components' model of addiction within a biopsychosocial framework. J Subst Use 2005; 10:191-197.

3. Lemmens JS, Valkenburg P, Peter J. Development and validation of a game addiction scale for adolescents. Media Psychol 2009; 12:77-95.

4. Petry NM, O'Brien CP. Internet gaming disorder and the DSM-5. Addiction 2013;108:1186-1187.

5. American Psychiatric Association. Diagnostic and Statistical Manual of Mental Disorders Fifth edition (DSM-5). Washington DC: American Psychiatric Publishing, 2013.

6. Petry NM, Rehbein F, Gentile DA, Lemmens JS, Rumpf HJ, Mossle T, et al. An international consensus for assessing Internet gaming disorder using the new DSM-5 approach. Addiction 2014; 109:1399-1406.

7. Saunders JB, Hao W, Long J, King DL, Mann K, Fauth-Buhler M, et al. Gaming disorder: its delineation as an important condition for diagnosis, management, and prevention. J Behav Addict 2017; 6:271-279.

8. World Health Organization, Gaming Disorder. 2019 https://www. who.int/features/qa/gaming-disorder/en/. Accessed September $15,2019$.

9. Aarseth E, Bean AM, Boonen H, Colder Carras M, Coulson M, Das D, et al. Scholars' open debate paper on the World Health Organization ICD-11 Gaming Disorder proposal. J Behav Addict 2017; 6:267-270.

10. Griffiths MD, van Rooij AJ, Kardefelt-Winther D, Starcevic V, Kiraly O, Pallesen S, et al. Working towards an international consensus on criteria for assessing Internet Gaming Disorder: a critical commentary on Petry et al. (2014). Addiction 2016; 111:167-175.

11. Kiraly O, Griffiths MD, Demetrovics Z. Internet gaming disorder and the DSM-5: Conceptualization, debates, and controversies. Curr Addict Rep 2015; 2:254-262.

12. Kiraly O, Bothe B, Ramos-Diaz J, Rahimi-Movaghar A, Lukavska $\mathrm{K}$, Hrabec $\mathrm{O}$, et al. Ten-Item Internet Gaming Disorder Test (IGDT-10): measurement invariance and cross-cultural validation across seven language-based samples. Psychol Addict Behav 2019; 33:91-103.

13. Kuss DJ, Griffiths MD, Pontes HM. Chaos and confusion in DSM-5 diagnosis of Internet Gaming Disorder: issues, concerns, and recommendations for clarity in the field. J Behav Addict 2017; 6:103-109.

14. van Rooij AJ, Prause N. A critical review of 'Internet addiction' criteria with suggestions for the future. J Behav Addict 2014; 3:203-213.

15. Demetrovics Z, Urban R, Nagygyorgy K, Farkas J, Griffiths $\mathrm{MD}$, Papay $\mathrm{O}$, et al. The development of the Problematic Online Gaming Questionnaire (POGQ). PLoS One 2012;7:e36417.

16. Huang Z, Wang M, Qian M, Zhong J, Tao R. Chinese Internet addiction inventory: developing a measure of problematic Internet use for Chinese college students. Cyberpsychol Behav 2007; 10:805-811.

17. Kiraly O, Sleczka P, Pontes HM, Urban R, Griffiths MD, Demetrovics Z. Validation of the Ten-Item Internet Gaming Disorder Test (IGDT-10) and evaluation of the nine DSM-5 Internet Gaming Disorder criteria. Addict Behav 2017; 64:253260.

18. Lemmens JS, Valkenburg PM, Gentile DA. The Internet Gaming Disorder Scale. Psychol Assess 2015; 27:567-582.

19. Pontes HM, Kiraly O, Demetrovics Z, Griffiths MD. The conceptualisation and measurement of DSM-5 internet gaming disorder: the development of the IGD-20 test. PLoS One 2014; 9:e110137.

20. Pontes HM, Griffiths MD. Measuring DSM-5 Internet gaming disorder: Development and validation of a short psychometric scale. Comput Human Behav 2015; 45:137-143. 
21. Rehbein F, Kleimann M, Mossle T. Prevalence and risk factors of video game dependency in adolescence: Results of a German nationwide survey. Cyberpsychol Behav Soc Netw 2010; 13:269277.

22. Pontes HM. Current practices in the clinical and psychometric assessment of internet gaming disorder in the era of the DSM-5: A mini review of existing assessment tools. Mental Health and Addiction Research 2016; 1:18-19.

23. van Rooij AJ, Schoenmakers TM, van de Mheen D. Clinical validation of the C-VAT 2.0 assessment tool for gaming disorder: a sensitivity analysis of the proposed DSM-5 criteria and the clinical characteristics of young patients with 'video game addiction.' Addict Behav 2017; 64:269-274.

24. Rehbein F, Kliem S, Baier D, Mossle T, Petry NM. Prevalence of Internet gaming disorder in German adolescents: diagnostic contribution of the nine DSM-5 criteria in a state-wide representative sample. Addiction 2015; 110:842-851.

25. Chiu YC, Pan YC, Lin YH. Chinese adaptation of the Ten-Item Internet Gaming Disorder Test and prevalence estimate of Internet gaming disorder among adolescents in Taiwan. J Behav Addict 2018; 7:719-726.

26. Mannikko $\mathrm{N}$, Ruotsalainen $\mathrm{H}$, Tolvanen $\mathrm{A}$, Kaariainen $\mathrm{M}$. Psychometric properties of the Internet Gaming Disorder Test (IGDT-10) and problematic gaming behavior among Finnish vocational school students. Scand J Psychol 2019; 60:252-260.

27. Evren C, Dalbudak E, Topcu M, Kutlu N, Evren B. The psychometric properties of the Turkish version of the Internet Gaming Disorder Scale. Dusunen Adam The Journal of Psychiatry and Neurological Sciences 2017; 30:316-324.

28. Evren C, Dalbudak E, Topcu M, Kutlu N, Evren B, Pontes HM. Psychometric validation of the Turkish nine-item Internet Gaming Disorder Scale-Short Form (IGDS9-SF). Psychiatry Res 2018; 265:349-354.

29. Arıcak OT, Dinc M, Yay M, Griffiths MD. Adapting the Short Form of the Internet Gaming Disorder Scale into Turkish: Validity and reliability. Addicta: The Turkish Journal on Addictions 2018; 5:615-636.
30. Demetrovics Z, Urban R, Nagygyorgy K, Farkas J, Zilahy D, Mervo B, et al. Why do you play? The development of the motives for online gaming questionnaire (MOGQ). Behav Res Methods 2011; 43:814-825.

31. Evren C, Evren B, Dalbudak E, Topcu M, Kutlu N. Psychometric validation of the Turkish Motives for Online Gaming Questionnaire (MOGQ). Addicta: The Turkish Journal on Addictions (In press).

32. Byrne BM. Structural Equation Modeling with AMOS: Basic Concepts, Applications and Programming. Second ed., New York: Taylor and Francis, 2010.

33. Hair JF, Black WC, Babin BJ, Anderson RE. Multivariate Data Analysis. Seventh ed., Upper Saddle River NJ: Prentice-Hall, 2010.

34. Cattell RB. The scree test for the number of factors. Multivariate Behav Res 1966; 1:245-276.

35. Ferguson E, Cox T. Exploratory factor analysis A user's guide. Int J Sel Assess 1993; 1:84-94.

36. Kaiser HF. The application of electronic computers to factor analysis. Educ Psychol Meas 1960; 20:141-151.

37. Lin CY, Luh WM, Cheng CP, Yang AL, Su CT, Ma HI. Measurement equivalence across child self-reports and parentproxy reports in the Chinese version of the Pediatric Quality of Life Inventory version 4.0. Child Psychiatry Hum Dev 2013; 44:583-590.

38. Wu TH, Chang CC, Chen CY, Wang JD, Lin CY. Further psychometric evaluation of the Self-Stigma Scale-Short: measurement invariance across mental illness and gender. PLoS One 2015; 10:e0117592.

39. Pontes HM, Macur M, Griffiths MD. Internet gaming disorder among Slovenian primary school children: findings from a nationally representative sample of adolescents. J Behav Addict 2016; 5:304-310.

40. Monacis L, Palo V, Griffiths MD, Sinatra M. Validation of the Internet Gaming Disorder Scale - Short-Form (IGDS9-SF) in an Italian-speaking sample. J Behav Addict 2016; 5:683-690. 